\title{
A novel, lactase-based selection and strain improvement strategy for recombinant protein expression in Kluyveromyces lactis
}

\author{
Jorrit-Jan Krijger ${ }^{1}$, Jan Baumann ${ }^{1}$, Melanie Wagner ${ }^{1}$, Katja Schulze ${ }^{1}$, Christian Reinsch ${ }^{1}$, Thomas Klose ${ }^{1}$, \\ Osita F Onuma', Claudia Simon², Sven-Erik Behrens ${ }^{2}$ and Karin D Breunig ${ }^{1 *}$
}

\begin{abstract}
Background: The Crabtree-negative yeast species Kluyveromyces lactis has been established as an attractive microbial expression system for recombinant proteins at industrial scale. Its $L A C$ genes allow for utilization of the inexpensive sugar lactose as a sole source of carbon and energy. Lactose efficiently induces the LAC4 promoter, which can be used to drive regulated expression of heterologous genes. So far, strain manipulation of K. lactis by homologous recombination was hampered by the high rate of non-homologous end-joining.
\end{abstract}

Results: Selection for growth on lactose was applied to target the insertion of heterologous genes downstream of the LAC4 promoter into the $K$. lactis genome and found to yield high numbers of positive transformants.

Concurrent reconstitution of the $\beta$-galactosidase gene indicated the desired integration event of the expression cassette, and $\beta$-galactosidase activity measurements were used to monitor gene expression for strain improvement and fermentation optimization. The system was particularly improved by usage of a cell lysis resistant strain, VAK367-D4, which allowed for protein accumulation in long-term fermentation. Further optimization was achieved by increased gene dosage of KIGAL4 encoding the activator of lactose and galactose metabolic genes that led to elevated transcription rates. Pilot experiments were performed with strains expressing a single-chain antibody fragment $\left(s c F v_{o x}\right)$ and a viral envelope protein (BVDV-E2), respectively. scFvox was shown to be secreted into the culture medium in an active, epitope-binding form indicating correct processing and protein folding; the E2 protein could be expressed intracellularly. Further data on the influence of protein toxicity on batch fermentation and potential post-transcriptional bottlenecks in protein accumulation were obtained.

Conclusions: A novel Kluyveromyces lactis host-vector system was developed that places heterologous genes under the control of the chromosomal LAC4 promoter and that allows monitoring of its transcription rates by $\beta$-galactosidase measurement. The procedure is rapid and efficient, and the resulting recombinant strains contain no foreign genes other than the gene of interest. The recombinant strains can be grown non-selectively in rich medium and stably maintained even when the gene product exerts protein toxicity.

\section{Background}

Kluyveromyces lactis is one of few yeast species that can utilize lactose as a sole source of carbon and energy. It can be isolated from dairy products and is used at industrial scale for preparation of the enzyme lactase ( $\beta$-galactosidase). $K$. lactis received considerable interest as a eukaryotic cell factory for heterologous protein

\footnotetext{
* Correspondence: karin.breunig@genetik.uni-halle.de

${ }^{1}$ Institute of Biology, Martin-Luther University Halle-Wittenberg, Halle 06120, Germany

Full list of author information is available at the end of the article
}

production due to physiological properties that distinguish it from Saccharomyces cerevisiae [1-3]. In particular, the Crabtree effect, which in S. cerevisiae leads to the repression of respiration under aerobic conditions, is absent or less pronounced in K. lactis allowing for high dilution rates and high biomass yields in fermentation processes [4-6].

With the recombinant expression of prochymosin in $K$. lactis, an industrial process was established the product of which is used in cheese manufacturing [1]. The approval of $K$. lactis-derived proteins in the food

\section{Biomed Central}


industry confirms the absence of biohazard in the host organism, which is an important aspect, for example, in the development of $K$. lactis-based oral vaccines that represents a major goal of our work [7].

A $K$. lactis-based expression system is commercially available that makes use of the strong, inducible $L A C 4$ promoter to drive the expression of heterologous proteins $[3,8] . L A C 4$ is one of the two $L A C$ genes, not found in $S$. cerevisiae, that are responsible for lactose assimilation. It encodes $\beta$-galactosidase and is divergently transcribed and co-regulated with the lactose permease gene $L A C 12$. The $L A C$ genes are located in a subtelomeric position, and polymorphic sites have been reported [9]. The LAC4 promoter, which has been extensively studied [10-12], is subject to transcriptional activation by KlGal4 (also termed Lac9), a homologue of the prototypic $S$. cerevisiae transactivator Gal4 [12-14]. KlGal4 is activated by intracellular galactose derived from cytosolic lactose hydrolysis or uptake of external galactose. Activation is controlled by a feedback regulatory network consisting of the KlGal4 inhibitor, KlGal80, and the bi-functional protein KlGal1 $[15,16]$. KlGal1 catalyses the conversion of galactose into galactose-1-phosphate, the first step in galactose assimilation [17]. In addition to this enzymatic activity, KlGal1 functions as an inducer of KlGal4 by binding to and inactivating KlGal80 [18].

Here we describe a novel strategy for stable, heterologous gene expression in $K$. lactis that makes use of KlGal4 to drive regulated or constitutive transcription and growth on lactose to select for integration of transgenes at the $L A C$ locus. In the described host-vector system, $\beta$-galactosidase activity parallels transcription of the transgene and can be used in the establishment of fermentation protocols and selection of strains with elevated transcription. In two pilot studies we demonstrate successful secretion of a single-chain antibody fragment $\left(\mathrm{scFv}_{\mathrm{ox}}\right)$ and intracellular production of a viral envelope protein, the E2 protein of bovine viral diarrhea virus (BVDV). Expression of viral antigens in food-grade yeast strains is a first step towards development of yeast-based oral vaccines. As described elsewhere recombinant $K$. lactis cells could be directly used for vaccination using the strategy reported here [Arnold et al, PLoS One, in press].

\section{Material and methods}

\section{K. Lactis strains}

Strain RUL1888 MATa ura3-59, a ura3 derivative of CBS2359 (NRRL Y-1140), was obtained from Dr. Yde Steensma (Leiden University, NL). RUL1888-D4 was generated by one-step gene disruption replacing $L A C 4$ sequences between +358 and +1181 by the ScURA3 gene. The antioxazolone single-chain antibody $\left(\mathrm{scFv}_{\mathrm{ox}}\right)$-producing strain RULOx was generated by replacing the ScURA3 gene with a $\mathrm{scFv}_{\mathrm{ox}}$ expression cassette by homologous recombination. Strain VAK367 metA ura3-5 was derived from CBS2359/ 152 [19] via two rounds of mutagenesis selecting for 5-fluoroorotic acid resistance and screening for high stability in stationary phase and killer-negative phenotype. VAK367-D4 is a lac4::ScURA3 derivative of VAK367, analogous to RUL1888-D4. VAK367-D4-derived recombinant strains are listed in Table 1.

\section{Plasmids}

Plasmid KIp3 is an integrative $K$. lactis vector for homologous integration at the LAC4 or lac4::ScURA3 locus. It consists of a ColE1 derived $E$. coli vector with the ampicillin resistance gene and an expression cassette based on the $L A C 4$ promoter and TEF1 terminator that are separated by a unique SalI site for insertion of genes of interest. The TEF1 terminator is followed by the KlGAL80 promoter and the 5 '-end of the LAC4 open reading frame. The cassette was generated by fusion-PCR in multiple steps

Table 1 Kluyveromyces lactis strains

\begin{tabular}{|c|c|c|c|c|}
\hline Name & Parent & Relevant genotype & Heterologous gene & Reference \\
\hline RUL1888 & CBS2356 & Klura3-59 & none & H.Y. Steensma (Univ. Leiden NL) \\
\hline RUL1888-D4 & RUL1888 & lac4::SCURA3 & SCURA3 & this work \\
\hline RULOx & RUL1888-D4 & $P_{L A C 4}-S C F V_{O X}$ & $S C F V_{O X}^{a}$ & this work \\
\hline RULOx/D802 & RULOx & 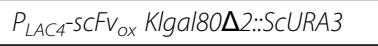 & SCURA3 & this work \\
\hline VAK367 & CBS 2359/152 & metA ura3-5 & none & this work \\
\hline VAK367-D4 & VAK367 & lac4::SCURA3 & SCURA3 & this work \\
\hline VAK726 & VAK367-D4 & $P_{L A C 4}-E 2-1_{B V D V}$ & $E 2-1_{B V D V}^{b}$ & this work \\
\hline VAK746 & VAK367-D4 & $P_{L A C 4}-E 2-1_{B V D V}$ Klgal80 $22:: S C U R A 3$ & $E 2-1_{B V D V}^{b}$ & this work \\
\hline VAK834 & 726VAK & $P_{L A C 4}-E 2-1_{B V D V}(\mathrm{pLI}-1)$ & $E 2-1_{B V D V}^{b}$ & this work \\
\hline VAK836 & VAK726 & $P_{L A C 4}-E 2-1_{B V D V}(\mathrm{pLI}-1)_{4}$ & $E 2-1_{B V D V}^{b}$ & this work \\
\hline VAK906 & VAK726 & $P_{L A C 4}-E 2 o p t(p L \mid-1)_{n}$ & E2-opt $t_{B V D V}^{c}$ & this work \\
\hline
\end{tabular}

${ }^{a}$ gene encoding oxazolon specific single-chain antibody fragment.

b E2-1 segment of the bovine viral diarrhea virus (strain CP7) containing the E2 ORF and $5^{\prime}$ and $3^{\prime}$ flanking sequences.

c yeast codon-optimized synthetic gene corresponding to VSV-E2(MTdel), which lacks the membrane anchor and is derived from BVDV strain NADL [22]. 
(Additional file 1). The cassette can be separated from the vector backbone by cleavage e.g. with $H p a \mathrm{I}$, which creates flanking regions of homology of 1 and $1.3 \mathrm{kbp}$, respectively. $\mathrm{KIpOx}$ is a derivative of KIp3 containing the S.cerevisiae alpha-factor prepro sequence fused to the $s c F v_{o x}$ sequence with a C-terminal c-myc tag (derived from plasmid pHEN1-1 [20]). KIp3-E2-1 [7] is a derivative of KIp3 containing the coding sequence of the C-terminus of BVDVE1 (including the signalase E1/E2cleavage site) and the full-length coding sequence of the BVDV-E2 (strain BVDV CP7, nucleotides 2273-3580; [21]; inserted as a SalI-XhoI fragment into the unique SalI site. In strain VAK906 this fragment was replaced by a yeast codon-optimized synthetic gene corresponding to VSV-E2(MTdel), which lacks the membrane anchor and is derived from BVDV strain NADL [22]. pLI-1 is an ScURA3-based integrative vector carrying the KlGAL4 gene (LAC9-1 allele) [23].

\section{Fermentation}

Fermentations were carried out in a DasGip parallel bioreactor system (DasGip AG, Jülich, Germany) with four $2 \mathrm{~L}$ fully equipped fermenters. For growth studies, parallel processes were operated in a batch mode with a working volume of $0.7 \mathrm{~L}$. YP medium ( $2 \%$ peptone, $1 \%$ yeast extract) was supplemented with mineral salts, methionine, uracil, adenine (20 mg/L each), and niacin (12 mg/L). Start lactose concentration was set to $30 \mathrm{~g} / \mathrm{L}$. The temperature of the yeast culture was maintained at $30^{\circ} \mathrm{C}$ and the dissolved oxygen level was controlled to $30 \%$ saturation. A $\mathrm{pH}$ value of 5.0 was controlled during the process via addition of $2 \mathrm{M} \mathrm{NaOH}$ and $2 \mathrm{M} \mathrm{H}_{3} \mathrm{PO}_{4}$. Samples were taken from the culture broth during the process to determine $\mathrm{OD}_{600}$, residual lactose and ethanol concentrations. One $\mathrm{OD}_{600}$ unit corresponds to $0.38 \mathrm{~g} / \mathrm{L}$ dry cell weight.

\section{Immunofluorescence microscopy}

$K$. lactis strain VAK726 was grown in YPLac medium ( $2 \%$ peptone, $1 \%$ yeast extract, $0.5 \%$ lactose), followed by formaldehyde fixation (5\% final concentration) for $3 \mathrm{~h}$ at $30^{\circ} \mathrm{C}$. After washing the cells with sorbitol buffer $(50 \mathrm{mM}$ HEPES; $1 \mathrm{M}$ sorbitol, $\mathrm{pH}$ 7.5), cell walls were degraded with cell wall degrading enzymes (zymolyase $10 \mathrm{mg} / \mathrm{ml}$ and glusulase, 1:4) for about $30 \mathrm{~min}$. Protoplasts were collected by centrifugation, and resuspended in sorbitol buffer, followed by permeabilization of the cell membrane with $0.1 \%$ Triton X-100 (in sorbitol buffer). $20 \mu \mathrm{l}$ of the suspension was applied to poly-L-lysine-coated object slides. The dried slides were washed 3 times in PBS. After blocking (3\% BSA; $0.1 \%$ cold fish gelatin, $0.1 \%$ Triton $\mathrm{X}-100 ; 0.05 \%$ Tween20) a $90 \mathrm{~min}$ incubation with a monoclonal anti-E2-antibody, [WB-214 (PA2020; c.c.pro, Germany); 1:100 in blocking solution] was performed, followed by 90 min incubation with a secondary antibody (Dylight 488, Dianova; 1:100 in blocking solution). In addition, cell nuclei and plasma membranes were stained with DAPI $(1 \mu \mathrm{g} / \mathrm{ml})$ and DiL C18 (Invitrogen; 1:1000 in $\mathrm{PBS})$, respectively. Fluorescence signals were recorded with a confocal laser scanning microscope (TCS SP5, Leica). DAPI fluorescence (absorption $358 \mathrm{~nm}$; emission $461 \mathrm{~nm}$ ) was excited with a DPSS Laser (405 nm); DyLight488 (absorption $493 \mathrm{~nm}$; emission $518 \mathrm{~nm}$ ) with an Argon Laser (488 nm) and DilC18 (absorption $549 \mathrm{~nm}$; emission $565 \mathrm{~nm}$ ) with a HeNe Laser (543 nm).

\section{Preparation of BSA-oxazolone}

Bovine serum albumin (BSA)-oxazolone conjugate was produced according to Mäkelä et al. [24]. Briefly, $75 \mathrm{mg}$ of oxazolon (4-Ethoxymethylene-2-phenyl-2-oxazolin-5-one; Sigma E-0753) was added to $20 \mathrm{ml}$ of $50 \mathrm{mg} / \mathrm{ml} \mathrm{BSA}$ (Albumin bovine Fraction V, pH 5.2, Serva 11922) in 5\% (w/v) $\mathrm{NaHCO}_{3}$ and shaken gently for $24 \mathrm{~h}$ at $4^{\circ} \mathrm{C}$. After centrifugation for $30 \mathrm{~min}$ at $30,000 \mathrm{xg}$, the supernatant was dialysed three times against $2 \mathrm{~L}$ of $0.15 \mathrm{M} \mathrm{NaCl}$ at $4{ }^{\circ} \mathrm{C}$ with gentle stirring, each step at least $12 \mathrm{~h}$. The concentration of oxazolone was calculated from the absorption at $352 \mathrm{~nm}$ of the dialysate, diluted with $0.15 \mathrm{M} \mathrm{NaCl}$ to give values between 0.1 and $0.6\left(\epsilon_{352}=32,000 \mathrm{M}^{-1} \mathrm{~cm}^{-1}\right)$. After determination of the BSA concentration, the coupling ratio was calculated and lay typically between 19 and 26 moles of oxazolone per mol of BSA with a final BSA concentration of $19-44 \mathrm{mg} / \mathrm{ml}$. The dialysate was supplemented with an equal volume of glycerol and stored at $-20^{\circ} \mathrm{C}$ in $100 \mu \mathrm{l}$ aliquots.

\section{ELISA for $\mathrm{scFv}_{\text {ox }}$ detection}

Microtiter plates (NUNC Maxisorp) were coated by filling the wells with $120 \mu \mathrm{l}$ of BSA-oxazolone conjugate, diluted 1:1000 in $0.1 \mathrm{M} \mathrm{NaCO}_{3} \mathrm{pH}$ 8.0. Filled plates were wrapped with parafilm and incubated overnight at $4^{\circ} \mathrm{C}$. After incubation, the plates were emptied by vigorous shaking, filled with $120 \mu \mathrm{l}$ of Blocking Reagent (Roche 1112 589) diluted 1:10 in distilled water and gently shaken on a vortex for $15 \mathrm{sec}$. This procedure was repeated three times. Finally the wells were filled with $120 \mu \mathrm{l}$ of Blocking Reagent and incubated at room temperature for 1.5 hours. Supernatants from $5 \mathrm{ml} K$. lactis cultures (WT and $\mathrm{scFv}_{\text {ox }}$-producing strains grown in YP with the indicated carbon sources at $120 \mathrm{rpm}$ and $30^{\circ} \mathrm{C}$ for up to 110 hours) were two-fold diluted serially (undiluted to 16-fold dilution). The samples were mixed with equal volumes of Blocking Reagent and $100 \mu$ l were filled in the wells of the blocked plate, followed by 1 hour incubation at room temperature. After incubation, the plates were washed as before, filled with $100 \mu \mathrm{l}$ of primary antibody (anti-c-myc antibody, Roche 1667 149), diluted 1:5000 in Blocking Reagent and incubated at room temperature for 1 hour. After washing, wells were filled with $100 \mu \mathrm{l}$ secondary antibody (anti mouse 
IgG-POD, Chemicon AP308P), diluted 1:2000 in Blocking Reagent and the plate was incubated at room temperature for 1 hour. After incubation, the plate was washed with substrate buffer $(3.25 \mathrm{mM}$ Na-perborate, $40 \mathrm{mM} \mathrm{Na}$ citrate, $60 \mathrm{mM} \mathrm{Na}_{2} \mathrm{HPO}_{4}, \mathrm{pH} 4.5$, stored at $4^{\circ} \mathrm{C}$ ) and filled with $100 \mu \mathrm{l}$ ABTS (Roche 1112 422, 1 tablet per $50 \mathrm{ml}$ of substrate buffer, stored at $4^{\circ} \mathrm{C}$ in darkness) per well. Reading was performed in a Tecan Sunsrise microplate reader, taking $405 \mathrm{~nm} / 450 \mathrm{~nm}$ difference readings with $1 \mathrm{~min}$ intervals for $20 \mathrm{~min}$ with $10 \mathrm{sec}$. shaking between readings. Amounts of $\mathrm{scFv}_{\mathrm{ox}}$ were determined by comparing $\triangle \mathrm{A} 405$ for the supernatant samples to serial dilutions of a reference $\mathrm{scFv}_{\text {ox }}$ preparation of known concentration, purified from Nicotiana tabacum [25].

\section{Dot blots for BVDV-E2 detection}

After growth for $8 \mathrm{~h}$ in YPLac medium, cells were harvested and protein extracts were prepared by glass bead disruption. $10 \mu \mathrm{l}$ of protein extracts, adjusted to the same concentration, were applied to a nitrocellulose membrane (GE Healthcare). After drying and blocking (2\% BSA in PBS) the membrane was incubated with an anti-E2-antibody (WB214; 1:100) for $90 \mathrm{~min}$, followed by incubation with the secondary infrared-labeled antibody (anti-ms-IRDy-800CW; LI-COR Biosciences, 1:500) for another $90 \mathrm{~min}$. Fluorescence signals were detected with an Odyssey infrared imaging system (LI-COR Bioscience). Excitation and emission wavelengths were $493 \mathrm{~nm}$ and $518 \mathrm{~nm}$, respectively.

\section{Northern blotting}

Yeast RNA was isolated by phenol-chloroform extraction as described [26]. $5 \mu \mathrm{g}$ total RNA was separated by agarose/formaldehyde gel electrophoresis ( $1 \%$ agarose; $1 \mathrm{x}$ MOPS buffer: 0.04 M MOPS, $10 \mathrm{mM}$ sodium acetate, 1 mM EDTA, pH 7; 3.7\% formaldehyde) and blotted to Hybond $^{\mathrm{TM}_{-} \mathrm{N}^{+}}$(GE Healthcare) membrane. After UVcross-linking, the membranes were subjected to hybridization with RNA probes generated by in vitro transcription with T7RNA polymerase (Fermentas). Template DNAs were generated by PCR amplification of E2 and LAC4 sequences with one primer introducing the T7 promoter sequence. Primer sequences were T7-E2-r (TAATACG A CTCACTATAGGGAGATTAGGACTCA GCGAAGTAAT C) and T-Tef REV (TGGAATTGTGAGCGGATAAC) for E2; LAC4-right_T7_Promoter (TAATACGACTCACTAT AGGACAGCTTCATCAACCCCGTAT) and LAC4Sonde Right (CTTGAACGCTTTGAGTGCAG) for LAC4.

\section{KIGAL4 copy number determination by qPCR}

Yeast chromosomal DNA was isolated and KlGAL4 DNA content was determined in strains VAK834 and VAK836 by qPCR using primers KIGAL4q-F (CAGCAAAG TG CAGGTGAATG) and KlGAL4q-R (AACTCCTTGCACC GTATGAC) for the KlGAL4 gene and ACT1qPCR-FWD
(ACGCTCCAAGAGCCGTCTTC) and ACT1qPCR-REV (TATCATCCCAGTTGGTAACG) for KlACT1, which served as the reference single copy gene. DNA amplification was recorded by SYBR-green fluorescence in an IQ5 real-time PCR system (BioRad). The copy number of KlGAL4 in VAK906 was shown by semi-quantitative PCR to be at least two but was not quantified precisely.

\section{Results and discussion}

\section{A $\beta$-galactosidase based selection strategy for stable} transformation of $K$. Lactis

Though multicopy episomal vectors are available for $K$. lactis, transformants often accumulate segregants without plasmid, even under selective conditions. This phenomenon is particularly pronounced with plasmids coding for proteins that exert a cytotoxic effect when expressed at high quantity in the yeast cell. To overcome this drawback and to generally improve $K$. lactis as a host system for heterologous protein expression, this study aimed at establishing a straight-forward procedure for the generation of stable, recombinant $K$. lactis strains. For a regulated and reproducible gene expression, these strains should carry an expression cassette integrated at a defined chromosomal position downstream of the LAC4 promoter. Additionally, the K. lactis recombinants should display characteristics that enable reproducible growth in an inexpensive medium and under non-selective conditions without loss of the expression cassette.

Along these lines, we generated a lactose-deficient mutant $K$. lactis strain in which the LAC4 gene was disrupted by the ScURA3 gene. Complementary, an integrative vector was constructed that permits the insertion of heterologous genes of choice between the $L A C 4$ promoter-5'UTR sequence and a transcription terminator, the latter of which is followed by the KlGAL80 promoter and the 5' portion of the LAC4 ORF (Figure 1). Cleavage of this plasmid with suitable restriction enzymes generates a fragment with 1 and $1.3 \mathrm{kbp}$ flanking regions that, via homologous recombination, can replace the lac4::ScURA3 disruption cassette and restore an intact LAC4 ORF. Regeneration of the intact LAC4 ( $\beta$-galactosidase) gene allows for growth on lactose as a sole carbon source and thus enables the selection of the desired recombinant strains. In a second screening step, the loss of the $\mathrm{Ura}^{+}$phenotype serves as a marker to distinguish between a true replacement event and an unintentional insertion of the entire plasmid downstream of the lac4::ScURA3 locus, which can also restore an intact LAC4 gene (see below). Typically, a transformation experiment with $5 \mu \mathrm{g}$ of cleaved plasmid DNA gave 20 to $50 \mathrm{Lac}^{+}$transformants $80 \%$ of which were Ura ${ }^{-}$.

In such correctly generated recombinant strains, the heterologous gene ends up at the subtelomeric $L A C$ locus. It is controlled by the natural $L A C 4$ promoter and 


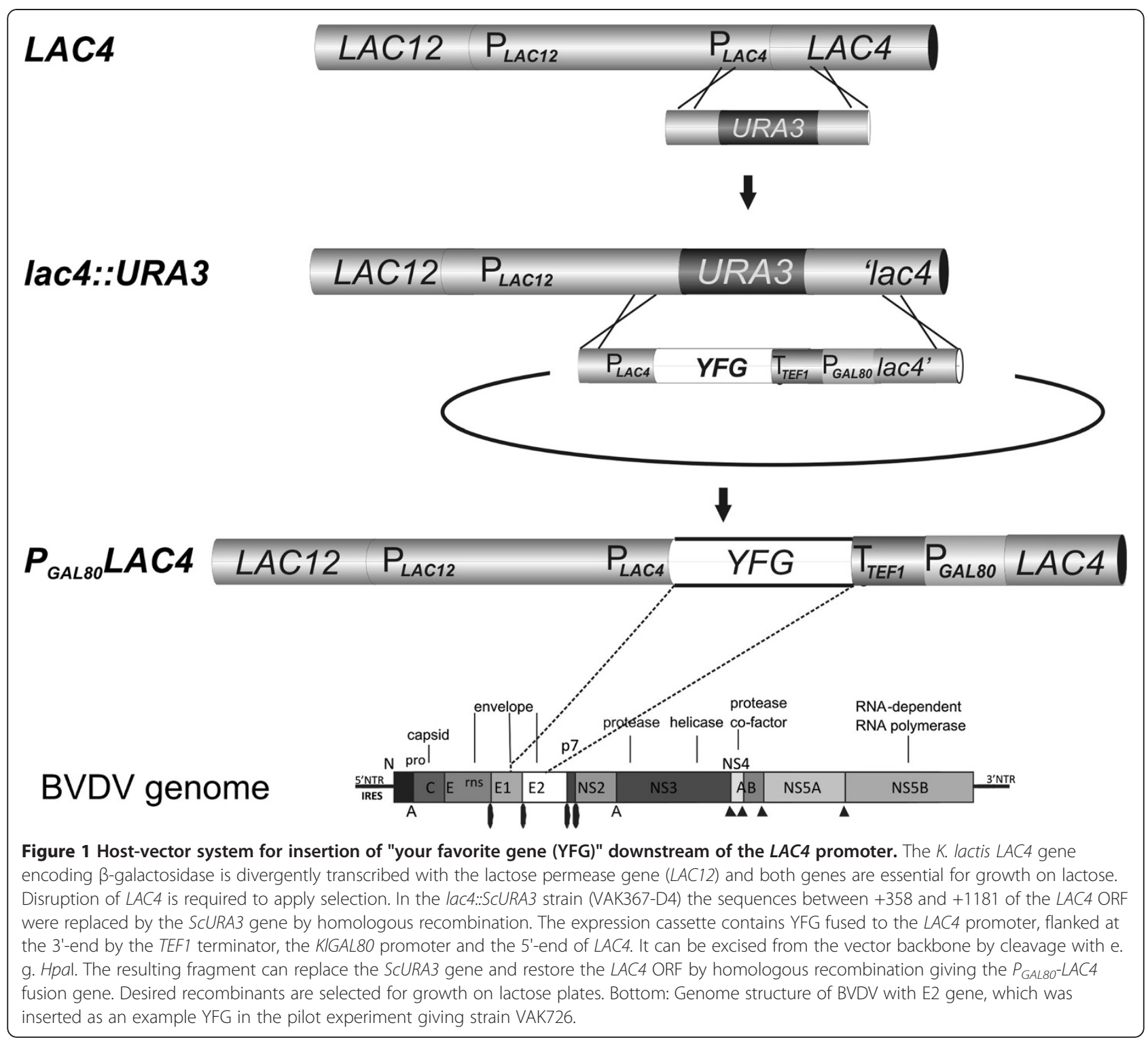

its long and complex control region, which co-regulates transcription of the divergent LAC4 and LAC12 genes [11]. Since expression of the LAC4 ORF is now under control of the weaker KlGAL80 promoter [27], the $\beta$-galactosidase levels produced by the recombinants are lower than that of the isogenic LAC4 parent strain. The use of the KlGAL80 promoter to drive $L A C 4$ expression accordingly serves two purposes: it avoids a tandem duplication of the LAC4 promoters, which could lead to the loss of the insert, and it allows using $\beta$-galactosidase activity as an indicator of heterologous gene transcription (see below).

\section{Expression studies with a secreted model protein}

The novel $K$. lactis host-vector system was next evaluated, first with a model gene encoding the single-chain antibody fragment anti-oxazolone-scFv with a C-terminal c-myc epitope $\left(\mathrm{scFv}_{\mathrm{ox}} ;[25]\right)$. To direct the recombinant protein to the secretory pathway, the $\mathrm{scFv}_{\text {ox }}$ coding gene was fused to the $S$. cerevisiae prepro-alpha factor sequence. The initial host strain was derived by LAC4 gene disruption as described [11] using strain RUL1888 (H.Y. Steensma, Univ. Leiden). After homologous recombination and selection of transformants on lactose plates, we screened for the Ura ${ }^{-}$phenotype and tested for integration of the expression cassette by PCR and Southern analysis. All transformants tested had restored the LAC4 gene, and the Ura' phenotype resulted, as expected, from the desired one-step gene replacement that brought the $s c F v_{o x}$ gene under the command of the $L A C$ control region. In these transformants, the $s c F v_{o x}$ gene represented YFG ("your favorite gene"; Figure 1) and was the only heterologous sequence in the 
K.lactis genome (strain RULOx). Alternatively, $\mathrm{Lac}^{+}$transformants that were still $\mathrm{Ura}^{+}$had restored the $L A C 4$ gene by integration of the entire plasmid with the expression cassette in single or multiple copies, and $\mathrm{scFv}_{\text {ox }}$ expression was driven by the truncated $L A C$ control region present on the transforming DNA (not shown).

To test for expression of the antibody fragment, several recombinants were pre-grown in glucose, shifted to lactose medium (time 0 ), and culture supernatants were analyzed by PAGE and Western blotting. A single band at $29 \mathrm{kDa}$, corresponding approximately to the expected size for the mature $\mathrm{scFv}_{\text {ox }}(28 \mathrm{kDa})$, was detected, indicating that the prepro-signal sequence had been removed and that the protein had passed through the secretory pathway. As expected, protein expression was inducible by lactose, confirming KlGal4-regulated transcription (Figure 2A). Consistently, knock-out of the KlGAL80 gene (strain RULOx/D802), which encodes the negative regulator of KlGal4, resulted in high expression levels of $s c F v_{o x}$ in the absence of the inducing sugars (Figure 2B).
ELISA experiments revealed that the heterologously expressed protein was delivered to the culture medium and bound the oxazolone antigen. The protein in the culture supernatant was quantified using a purified $\mathrm{scFv}_{\text {ox }}$ myc reference protein produced in Nicotiana tabacum (kindly provided by Dr. U. Conrad) as a standard. The specific binding activity of the yeast derived protein was identical to the one purified from plant cells. This indicates that $K$. lactis produced correctly folded $\mathrm{scFv}_{\mathrm{Ox}}$ and the affinity for the antigen was not affected by yeast specific modifications.

As described earlier, due to incomplete inactivation of the KlGal4 inhibitor under inducing conditions [27], higher $L A C 4$-controlled protein levels could be obtained in the Klgal80 mutant than in the induced wild type parent. In a first, non-optimized fermentation process of strain RULOx/D802 up to $4.4 \mathrm{mg} / \mathrm{liter} \mathrm{scFv}_{\text {ox }}$ protein was obtained with a maximum at 48 hours of batch cultivation (Figure 2C). In glucose the level of $\mathrm{scFv}_{\text {ox }}$ was about 2-3 fold higher than in glycerol grown cells. In

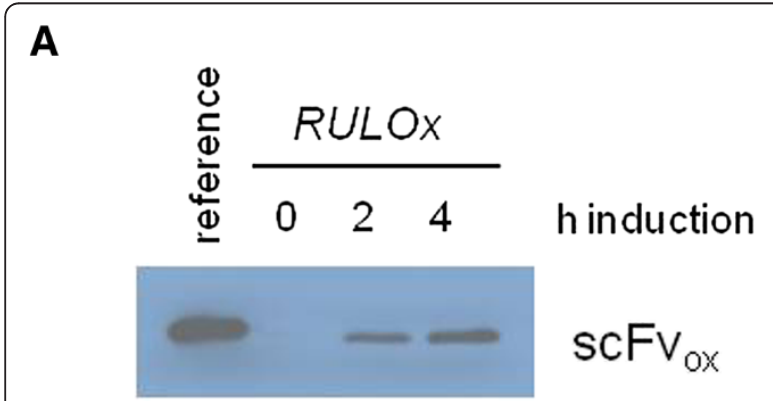

B
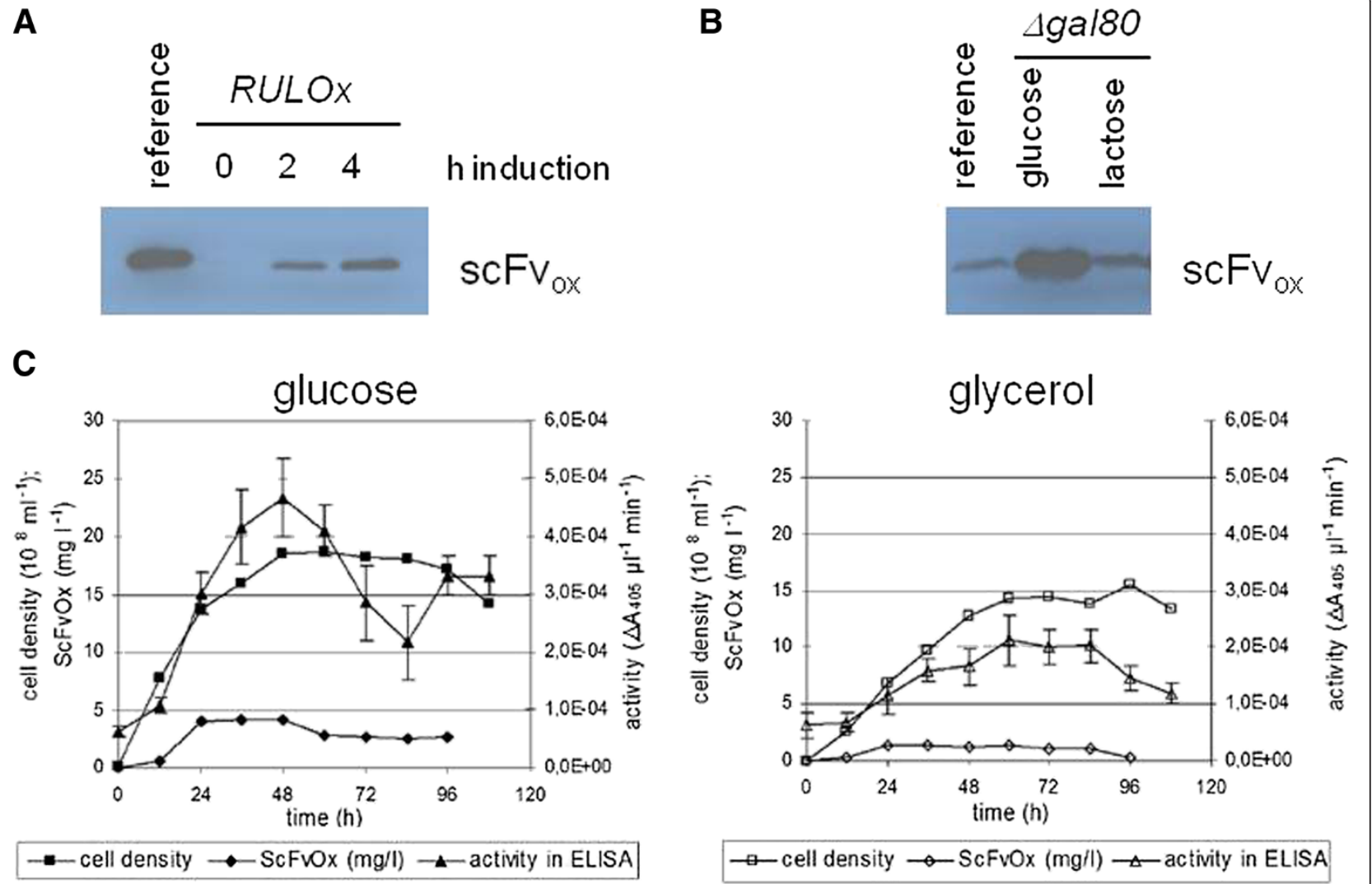

Figure 2 Analysis of production of anti-oxazolone single-chain antibody fragment $\mathbf{s c F v}_{\mathbf{O x}}$. A) Inducible expression of $s c F v_{0 x}$ in $s t r a i n$ RULOx. Soluble scFvox in supernatants of YNB-cultures pregrown in glucose, 0, 2 and 4 hrs after induction by lactose was detected by Western blotting. The reference lane contains $15 \mathrm{ng}$ of purified scFvox. B) Constitutive expression of scFvox in strain RULOx/D802 (KIgal80 $\Delta$ ). Upon disruption of the KIGAL80 gene higher levels of soluble $s c F v_{\text {ox }}$ were present in supernatants of glucose- than lactose-grown cells. The reference lane contains $5 \mathrm{ng}$ of purified scFvox. C) Long-term cultivation in glucose or glycerol medium ( $2 \%$ and $3 \%$ initial concentration, respectively) of the Klgal80 mutant strain expressing scFvox (RULOx/D802 strain). Cell density (squares) is plotted with oxazolone-binding activity (triangles) and protein content (diamonds) in culture supernatant as determined by ELISA and SDS-PAGE, respectively, against the pure reference protein. 
both media there was a good correlation between the accumulation of protein and binding activity. However, for unknown reasons, the binding activity dropped at late times in the batch process, while the protein appeared to be stable in Western analysis.

We had no evidence for intracellular protein accumulation or cell toxicity exerted by $\mathrm{scFv}_{\mathrm{ox}}$. Compared to Pichia pastoris, an established yeast host system for high level single chain antibody expression [28-31], K. lactis did not reach the high cell densities and productivity in our nonoptimized system. However, we have reason to believe that considerable improvement can be achieved by optimizing fermentation protocols and strain backgrounds. A very promising aspect of the $K$. lactis system is the low level of proteinase secretion and the solubility of the $\mathrm{scFv}_{\text {ox }}$.

With strain RULOx/D802 (Klgal80) Coomassie staining of culture supernatants revealed an unexpectedly large number of additional proteins. Moreover, $\beta$-galactosidase activity could be detected in the culture medium (not shown) at late time points of the cultivation, indicating cell lysis and release of cytoplasmic enzymes. The strain RULOx did not differ from the Klgal80 mutant derivative in this respect (except that $\beta$-galactosidase activity was much lower). Cell lysis occurred in the stationary phase and was also detected in the non-recombinant parent strain RUL1888 (Figure 3A), ruling out that the instability was caused by expression of the heterologous protein.

In fact, among a series of $K$. lactis wild type and laboratory strains that were subsequently screened, the majority showed extracellular $\beta$-galactosidase indicating that in many $K$. lactis strains cytosolic proteins are released by cell lysis. Crossing the RUL1888 strain to JA6 [32], a stable strain with 1000- fold lower extracellular $\beta$-galactosidase activity than RUL1888, resulted in diploids that behaved like the RUL1888 parent suggesting that the cell lysis phenomenon was dominant (Figure $3 \mathrm{~B}$ ). A high correlation was observed between high $\beta$-galactosidase activity in the culture supernatant and high protein content in the growth medium. We thus used this simple enzymatic assay to generate an alternative $K$. lactis host strain for heterologous protein expression, VAK367, that did not show significant cell lysis. VAK367 originated from a Ura derivative of CBS2359/152 [19]; it had undergone two rounds of mutagenesis and surprisingly was much more stable than the grandparent CBS2359 (data not shown). Disruption of the LAC4 gene in VAK367 using the lac4::ScURA3 knock-out cassette (see above) did not change this property (data not shown). Thus, the resulting strain, VAK367-D4 met ura3 lac4::ScURA3, was subsequently used as our standard host for the integration of heterologous genes.

\section{Expression of the E2 envelope protein of bovine viral diarrhea virus (BVDV)}

The above described selection and integration strategy was used to express several viral structural proteins. As described elsewhere, highly stable virus-like particles could be produced upon expression of murine polyoma virus VP1 in a Klgal80 mutant strain (Simon et al., submitted). Here we report a pilot experiment on the expression of BVDV-E2 (termed E2 in this text), a highly antigenic envelope protein of the important animal pathogen bovine viral diarrhea virus (BVDV). For this purpose, the BVDV ORF encoding the C-terminus of the envelope protein E1 and the entire E2 was amplified

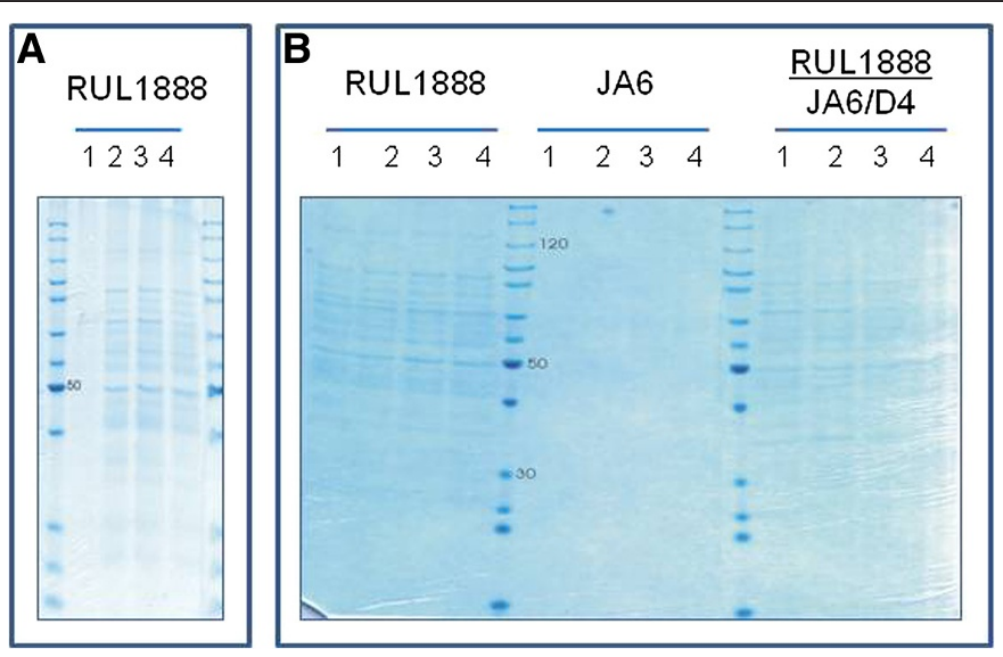

Figure 3 Leakage of $\boldsymbol{K}$. lactis proteins into the culture medium. A) Strain RUL1888 was grown for 6 (lane 1), 24 (lane 2), 30 (lane 3) and 48 hrs (lane 4) in YP medium with an initial concentration of 0.04\% galactose. B) Strain RUL1888, JA6 and a diploid strain resulting from a cross between JA6/D4 and RUL1888 were grown for 24 (lane 1), 48 (lane 2), 72 (lane 3) and 96 hrs (lane 4) in 1\% galactose. Culture supernatants were applied to SDS-PAGE and gels were stained after electrophoresis by Coomassie brilliant blue. 
from a cDNA of the viral isolate CP7 [21] and cloned into the KIp3 vector (E2-1 allele). An endogenous signal sequence and signal peptidase cleavage-site in the E1 portion should direct the expressed protein to the ER-membrane and enable correct processing of the E2 $\mathrm{N}$-terminus [33]. After cleavage of the plasmid with HpaI, which releases the expression cassette, transformation into VAK367-D4 and selection for growth on lactose were performed. The E2-1 allele was successfully integrated at the LAC4 locus (Figure 1, bottom line) as verified by PCR and Southern blotting (data not shown). The resulting strain was named VAK726.

Expression of the E2 protein and its intracellular localization were analyzed by immunofluorescence in intact yeast cells grown on lactose medium (Figure 4). These data indicated association of the recombinant E2 with intracellular membranes, most likely of the endoplasmic reticulum (ER). Further experiments are needed to confirm E2 localisation.

\section{Regulated versus constitutive expression of E2}

As shown above for $\mathrm{scFv}_{\text {ox }}$ and elsewhere for the polyoma VP1 (Simon et al., submitted), higher protein levels were obtained in a Klgal80 mutant conferring constitutive expression from the LAC4 promoter. Likewise, when the KlGAL80 gene of VAK726 was disrupted the resulting Klgal80 mutant, strain VAK846, displayed higher E2-1 mRNA levels than the induced parent strain with a high correlation between E2-1 and LAC4 mRNA levels (Figure 5). On the downside, the Klgal80 mutation was found to reduce the fitness of the strain $K$. lactis VAK746. That is, on $\beta$-galactosidase indicator plates containing X-Gal (5-bromo-4-chloro-indolyl-galactopyranoside) white colonies were repeatedly observed with this strain. PCR analysis of these colonies revealed that they had lost the expression cassette as well as the downstream LAC4 gene. We assumed that these variants were under positive selection due to the toxicity of the heterologous protein product when produced constitutively and at high
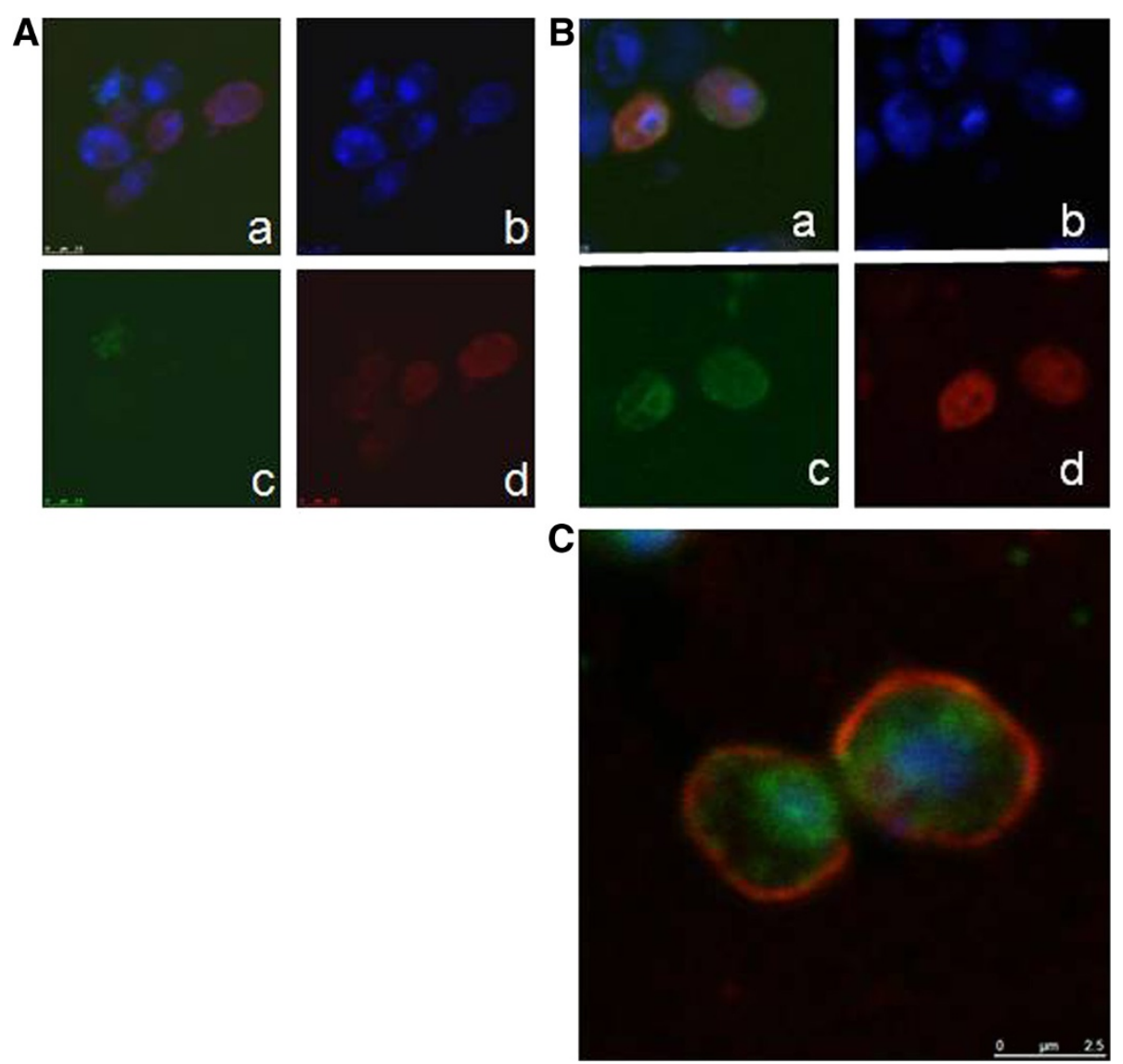

Figure 4 Detection of E2 protein expression in yeast cells by immunocytology. K. lactis strains VAK367 (A) and VAK726 (B and C), which contains the BVDV-E2 ORF in single copy under control of the LAC4 promoter, were grown under inducing conditions ( $2 \%$ lactose) and analyzed by confocal microscopy after staining with DAPI (panels b), E2-specific antibody WB-214 (PA2020; c.c.pro, Germany) and a polyclonal secondary antibody (Dylight 488; Dianova) (panels c) and Dil18 (Invitrogen) for staining of the plasma membrane (panels d). E2 and nuclear (DAPI) staining is merged in panels a. C) Overlay of DNA, membrane-, and E2- staining reveals accumulation of E2 in perinuclear regions suggesting localization in the ER. 


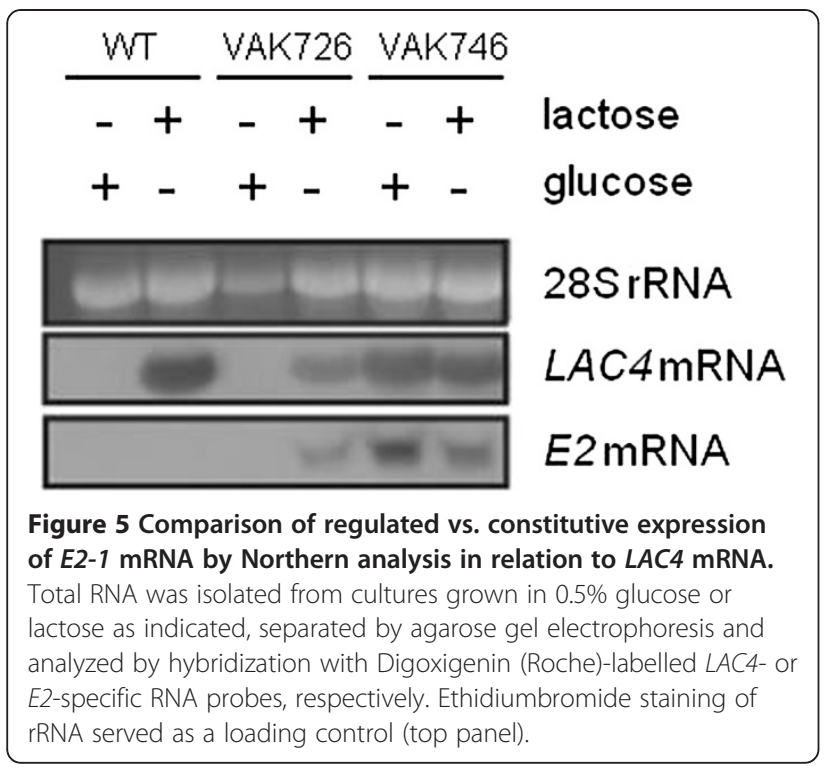

amounts in the yeast cell. We therefore decided to stick to a regulated expression of the E2 protein.

\section{Increased KIGAL4 gene dosage causes elevated expression}

A major advantage of the described genomic integration strategy is that the transcription regulation of the heterologous gene and of the $\beta$-galactosidase gene follows similar rules. Considering that transcription mediated by the LAC4 as well as the KlGAL80 promoter is elevated at higher concentrations of KlGal4 [23,34,35], we next tested whether E2 expression could be shifted by increasing the KlGAL4 gene dosage.

Accordingly, a KlGAL4 containing plasmid, pLI-1 [23], was integrated at the KlGAL4 locus of VAK726, and $\mathrm{Ura}^{+}$transformants were screened for elevated $\beta$-galactosidase activity on X-Gal-containing glucose plates. Interestingly, blue staining on glucose turned out to be a reliable marker for multiple integration of the KlGAL4 plasmid, probably due to an imbalance between KlGal4 and KlGal80. Tandem arrangement of the KlGAL4 gene copies was verified by PCR, and the gene copy number was determined by qPCR. Two strains containing 2 (strain VAK834) and 5 copies (strain VAK836) of the KlGAL4 gene were further analyzed. E2-1 mRNA levels were two and six-fold higher in strains VAK834 and VAK836, respectively, compared to VAK726 and showed a high correlation with $L A C 4$ mRNA levels (Figure 6).

To compare E2 protein levels in these yeast strains we established a dot-blot assay (Figure 7A) since denatured E2 was undetectable with the available antibodies. E2 protein present in BVDV-infected cell lysates served as a reference. Quantification of signal intensities in the dotblot assay indicated a $20 \%$ increase of the protein level

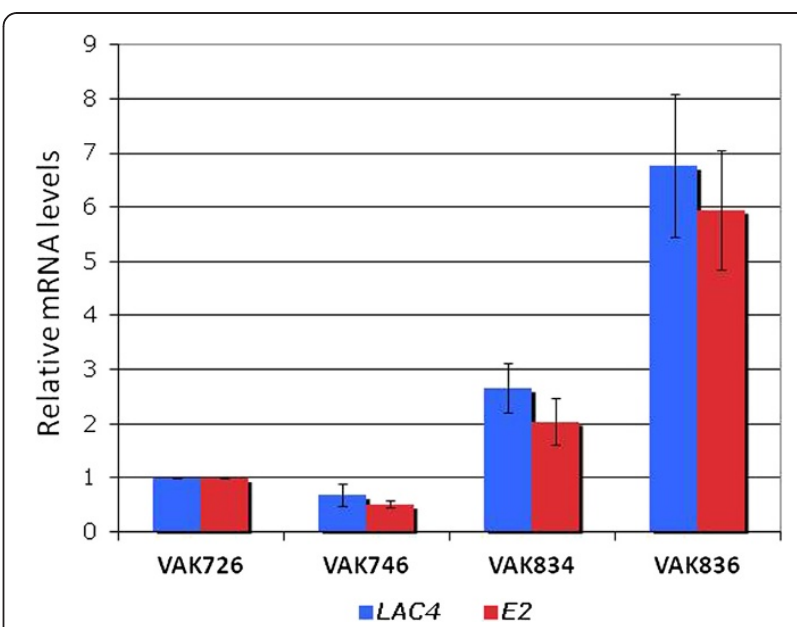

Figure 6 Influence of elevated KIGAL4 gene dosage on E2 and LAC4 mRNA levels. The E2-1-expressing strains VAK834 and VAK836 (Table 1) contain one and four tandem copies, respectively, of plasmid pLI-1 carrying KIGAL4 in addition to the endogenous KIGAL4 gene copy. mRNA was prepared from cells grown in $0.5 \%$ lactose and quantified by RT-qPCR using KIACT1 as a reference.

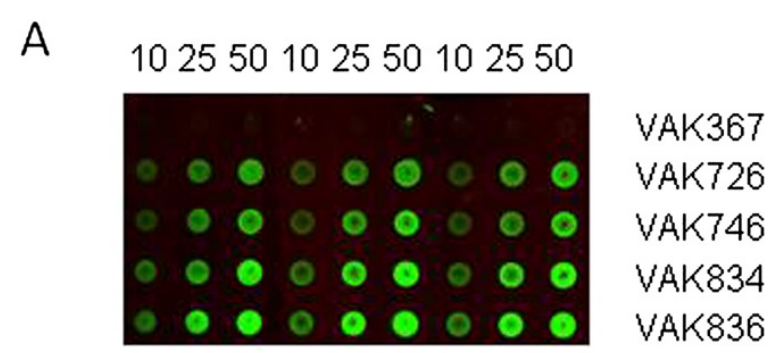

B

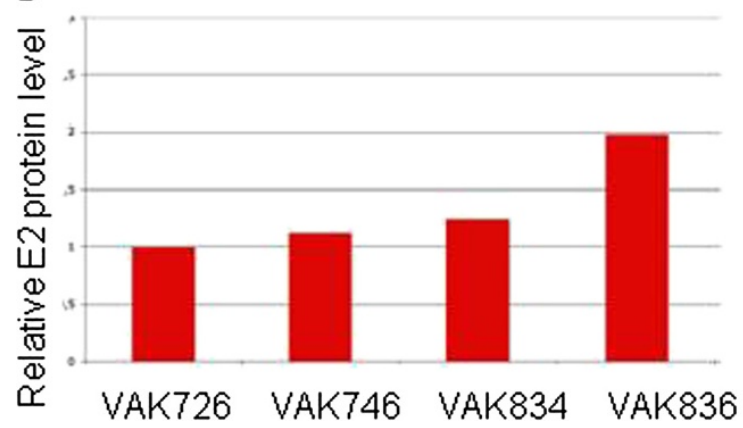

Figure 7 Quantification of E2 protein levels in different congenic $K$. lactis strains. A) Dot blots performed in triplicates with 10, 25 and $50 \mu \mathrm{g}$ of crude cell lysates from parent strain VAK367, the E2-producing strain VAK726; the Klgal80 mutant derivative of VAK726 (VAK746); and the two derivatives of VAK726 carrying two (VAK834) and five (VAK836) copies of the KIGAL4 gene, respectively. Cell lysates were applied to nitrocellulose membranes and incubated with E2-specific antibody WB-214 (PA2020; c.c.pro, Germany) and polyclonal secondary antibody (Dylight 488; Dianova). B) Relative fluorescence signal intensities were quantified as described in Material and Methods and are given relative to strain VAK726. 
in the two-copy strain and a two-fold increase in the five-copy strain (Figure 7B). Thus, the increase in transcript levels only partially translates into higher protein levels suggesting limitations at the level of translation or protein stability. We have clear indications that codon bias is one important factor limiting translation of viral genes in $K$. lactis [Arnold et al., in revision]. The expression of yeast codon optimized synthetic genes can circumvent this problem.

\section{Influence of elevated KIGAL4 gene dosage on fermentation of E2 production strains}

The two E2 expressing strains VAK726 and VAK834 were compared in batch fermentation with an initial lactose concentration of $3 \%$. The additional KlGAL4 gene copy in VAK834 caused a significantly higher growth rate in the exponential phase (Figure 8). However, this was associated with lower yield and increased ethanol production (Table 2). At later time points strain VAK726 overtook VAK834 and after 26 hours had accumulated higher biomass levels.

Another E2 production strain, VAK906, which contains at least two KlGAL4 gene copies, showed a different behavior. It resembles VAK834 in the initial fermentation phase but does not level off later. This indicates that the lower biomass accumulation of VAK834 is not due to the elevated KlGal4 concentration per se. Rather, the increased expression of the E2-1 protein appears to exert protein toxicity not found in VAK906. The E2 gene in VAK906 was
Table 2 Comparison of the fermentation process in three different E2 expression strains

\begin{tabular}{llllc}
\hline & $\boldsymbol{\mu}\left[\mathbf{h}^{-1}\right]^{\mathrm{a}}$ & $\mathbf{Y}_{\mathbf{x s}}[\mathbf{g} / \mathbf{g}]^{\mathrm{b}}$ & $\mathbf{Y}_{\mathrm{Ex}}[\mathbf{g} / \mathbf{g}]^{\mathrm{c}}$ & $\mathbf{X}_{\mathbf{f}}[\mathbf{g} / \mathbf{L}]^{\mathrm{d}}$ \\
\hline VAK726 & 0.21 & 0.24 & 0.29 & 7.6 \\
\hline VAK834 & 0.25 & 0.13 & 1.13 & 5.1 \\
\hline VAK906 & 0.27 & 0.15 & 0.84 & 10.7
\end{tabular}

${ }^{\mathrm{a}} \mu$, specific growth rate in exponential phase; ${ }^{\mathrm{b}} \mathrm{Y}_{\mathrm{XS}}$, biomass yield from substrate; ${ }^{\circ} Y_{E X}$, ethanol per biomass; ${ }^{d} X_{f}$, final biomass (after 26 hrs).

derived from another viral isolate and deviates in several aspects from the E2-1 allele present in VAK726 and VAK834 (see Material and methods). It remains to be clarified which property favors the performance in fermentation. The results demonstrate the benefit that is eventually gained from moderate KlGal4 overexpression. However, very high levels of the transactivator exert toxicity even in a strain containing no heterologous genes [36].

\section{Conclusions}

Here we present a new strategy to efficiently target heterologous genes to a specific location in a $K$. lactis chromosome for expression under the $K$. lactis LAC4 promoter. It differs from established expression systems in the following aspects:

(i). About $80 \%$ of the transformants selected on lactose medium are $\mathrm{Ura}^{-}$and have replaced the lac4:: ScURA3 disruption cassette by the transgene under the control of the complex LAC4 promoter despite

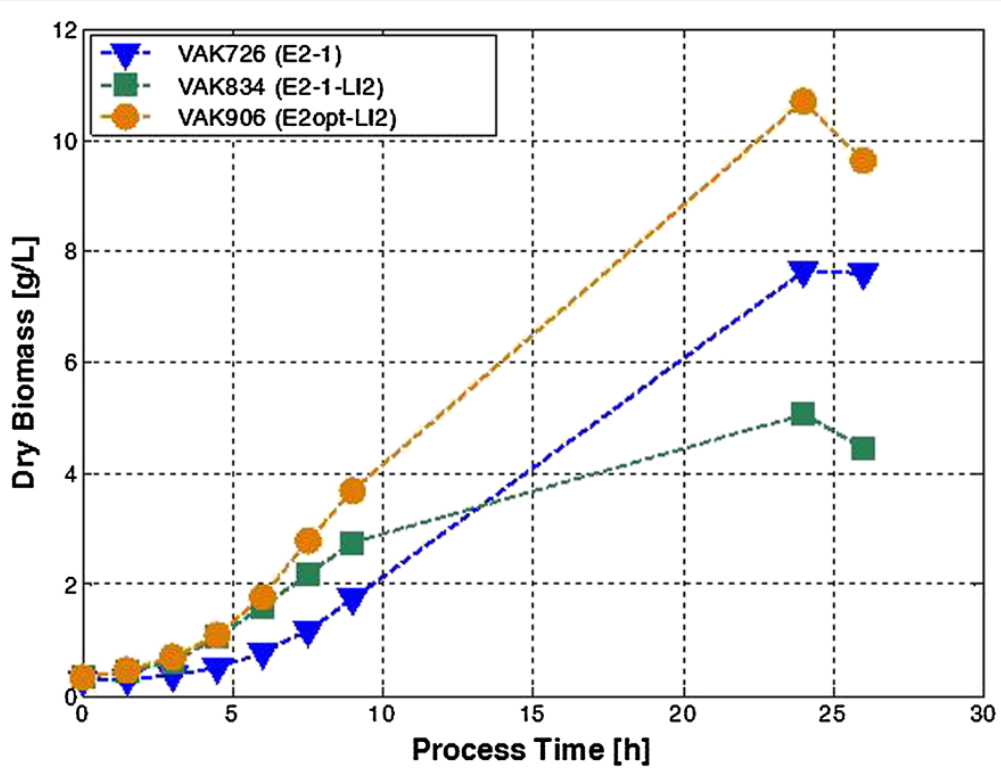

Figure 8 Comparison of biomass accumulation of three E2 protein expressing strains. Batch fermentation in supplemented YP-lactose medium (3\% initial concentration) was performed in a DasGip parallel bioreactor system (DasGip AG, Jülich, Germany) under controlled conditions (see Material and Methods). Biomass accumulation is plotted against process time. VAK726 and VAK834 express the E2-1 allele of BVDV strain CP7, VAK906 contains an E2 allele from BVDV strain NADL [22]. VAK834 and VAK906 carry multiple copies of the KIGAL4 transactivator gene. 
the commonly low frequency of homologous recombination in $K$. lactis.

(ii). No heterologous marker genes are used for selection of transformants; instead selection depends on reconstitution of the $K$. lactis $\beta$-galactosidase gene in a lac4 mutant background allowing for growth on lactose. Thus, the gene of interest is the only heterologous gene in the resulting recombinant strain.

(iii). There is no need for a 5 -fluoroorotic acid (FOA) selection step, which may result in cryptic mutations, to select for replacement of the lac4:: ScURA3 disruption. Since lac4 disruptions can be easily generated using dominant markers and X-Gal indicator plates for mutant selection, any prototrophic host strain, including polyploid industrial production strains, can be engineered to meet the requirements of this host-vector system.

(iv). Expression of $\beta$-galactosidase is driven by the KlGAL80 promoter, the activity of which parallels $L A C 4$ promoter activity. This allows optimizing fermentation protocols and strain improvement by simple established enzymatic assays.

(v). The recombinant strains are stable even when the gene product exerts cytotoxicity and can be grown under non-selective conditions in complex media if desired.

With the regulated production of an anti-oxazolone single-chain antibody and the bovine viral diarrhea virus envelope protein E2 we further confirmed the suitability of $K$. lactis as a eukaryotic microbial host for secretion and intracellular production of heterologous proteins that are otherwise difficult to express recombinantly. The inducible $L A C 4$ promoter can easily be converted into a strong constitutive promoter by gene disruption of the transcription inhibitor KlGal80 but, depending on the introduced heterologous gene, protein toxicity may become an issue. We demonstrated that inducible LAC4 promoter activity and expression of the transgene could be further improved by integration of additional copies of the KlGAL4 transcription activator gene. However, in the case of the BVDV-E2-1 allele cell viability dropped from $>95 \%$ to $<65 \%$ with elevated KlGAL4 gene dosage (data not shown). Moreover, elevated transcription rates did not directly correlate with protein accumulation and post-transcriptional steps were uncovered as potential bottlenecks. The regulated expression under control of the LAC4 promoter nevertheless allowed obtaining high recombinant protein expression levels.

\section{Additional file}

Additional file 1: Construction of the scFv.ox expression cassette.
Competing interests

The authors declare that they have no competing interests.

\section{Authors contributions}

JJK constructed the expression cassette and performed the scFvox experiments, JB and MW performed the E2 pilot experiment, CR analysed the cell lysis phenomenon, OFO and TK generated E2 expressing strains, KS and CS contributed to yeast fermentation, SEB designed E2 expression and Cosupervised the E2 pilot study, KDB conceived the strategy, supervised the work and drafted the manuscript. All authors read and approved the final manuscript.

\section{Acknowledgment}

This work was supported by BMBF funding "Innovative Regionale Wachstumskerne", project 03WKB01D and ForMaT program, project "Vakzinova" (03FO1232). We gratefully acknowledge technical support by Jenny Wieczorek and material provided by Prof. Dr. Uwe Conrad, IPK Gatersleben ( $\mathrm{sFV}_{\mathrm{Ox}}$ ) and Prof. Georg Herrler, VMH, Hannover (E2).

\section{Author details}

${ }^{1}$ Institute of Biology, Martin-Luther University Halle-Wittenberg, Halle 06120, Germany. ${ }^{2}$ Institute of Biochemistry and Biotechnology, Martin-Luther University Halle-Wittenberg, Halle 06120, Germany.

Received: 20 December 2011 Accepted: 17 May 2012

Published: 20 August 2012

\section{References}

1. Van den Berg JA, Van der Laken KJ, van Ooyen AJ, Renniers TC, Rietveld K, Schaap A, Brake AJ, Bishop RJ, Schultz K, Moyer D: Kluyveromyces as a host for heterologous gene expression: expression and secretion of prochymosin. Biotechnol (N.Y.) 1990, 8:135-139.

2. Gellissen G, Hollenberg CP: Application of yeasts in gene expression studies: a comparison of Saccharomyces cerevisiae, Hansenula polymorpha and Kluyveromyces lactis - a review. Gene 1997, 190:87-97.

3. van Ooyen AJ, Dekker P, Huang M, Olsthoorn MM, Jacobs DI, Colussi PA, Taron $\mathrm{CH}$ : Heterologous protein production in the yeast Kluyveromyces lactis. FEMS Yeast Res 2006, 6:381-392.

4. Mulder W, Scholten IHJM, Grivell LA: Carbon catabolite regulation of transcription of nuclear genes coding for mitochondrial proteins in the yeast Kluyveromyces lactis. Curr Genet 1995, 28:267-273.

5. Breunig KD, Bolotin-Fukuhara M, Bianchi MM, Bourgarel D, Falcone C, Ferrero I, Frontali L, Goffrini P, Krijger JJ, Mazzoni C, Milkowski C, Steensma HY, Wesolowski-Louvel M, Zeeman AM: Regulation of primary carbon metabolism in Kluyveromyces lactis. Enzyme Microb Technol 2000, 26:771-780.

6. Gonzalez-Siso Ml, Garcia-Leiro A, Tarrio N, Cerdan ME: Sugar metabolism, redox balance and oxidative stress response in the respiratory yeast Kluyveromyces lactis. Microb Cell Fact 2009, 8:46.

7. Breunig KD, Behrens SE: Procedure for oral vaccination against virus infection by means of recombinant yeast cells expressing viral protein. International application No. PCT/DE 2009/001623.

8. Read JD, Colussi PA, Ganatra MB, Taron CH: Acetamide selection of Kluyveromyces lactis cells transformed with an integrative vector leads to high-frequency formation of multicopy strains. Appl Environ Microbiol 2007, 73:5088-5096.

9. Naumov Gl: Identification of the lactose $L A C$ gene superfamilies in Kluyveromyces yeast. Dokl Biochem Biophys 2008, 420:158-160.

10. Leonardo JM, Bhairi SM, Dickson RC: Identification of Upstream Activator Sequences That Regulate Induction of the $\beta$-Galactosidase Gene in Kluyveromyces lactis. Mol Cell Biol 1987, 7:4369-4376.

11. Gödecke A, Zachariae W, Arvanitidis A, Breunig KD: Coregulation of the Kluyveromyces lactis lactose permease and $\beta$-galactosidase genes is achieved by interaction of multiple LAC9 binding sites in a $2.6 \mathrm{kbp}$ divergent promoter. Nucleic Acids Res 1991, 19:5351-5358.

12. Ruzzi M, Breunig KD, Ficca AG, Hollenberg CP: Positive regulation of the $\beta$-galactosidase gene from Kluyveromyces lactis is mediated by an upstream activation site that shows homology to the GAL upstream activation site of Saccharomyces cerevisiae. Mol Cell Biol 1987, 7:992-997.

13. Salmeron JM, Johnston SA: Analysis of the Kluyveromyces lactis positive regulatory gene $L A C 9$ reveals functional homology to, but sequence 
divergence from, the Saccharomyces cerevisiae GAL4 gene. Nucleic Acids Res 1986, 14:7767-7781.

14. Wray LVJ, Witte MW, Dickson RC, Riley MI: Characterization of a positive regulatory gene, $L A C 9$, that controls induction of the lactose-galactose regulon of Kluyveromyces lactis: structural and functional relationships to GAL4 of Saccharomyces cerevisiae. Mol Cell Biol 1987, 7:1111-1121.

15. Breunig KD: Regulation of transcription activation by Gal4p. Food technol biotechnol. 2000, 38:287-293.

16. Anders A, Lilie H, Franke K, Kapp L, Stelling J, Gilles ED, Breunig KD: The galactose switch in Kluyveromyces lactis depends on nuclear competition between Gal4 and Gal1 for Gal80 binding. J Biol Chem 2006, 281:29337-29348.

17. Meyer J, Walker-Jonah A, Hollenberg CP: Galactokinase encoded by GAL1 is a bifunctional protein required for induction of the GAL genes in Kluyveromyces lactis and is able to suppress the gal3 phenotype in Saccharomyces cerevisiae. Mol Cell Biol 1991, 11:5454-5461.

18. Zenke FT, Engels R, Vollenbroich V, Meyer J, Hollenberg CP, Breunig KD: Activation of Gal4p by galactose-dependent interaction of galactokinase and Gal80p. Science 1996, 272:1662-1665.

19. Wesolowski-Louvel M, Tanguy-Rougeau C, Fukuhara H: A nuclear gene required for the expression of the linear DNA-associated killer system in the yeast Kluyveromyces lactis. Yeast 1988, 4:71-81.

20. Hoogenboom HR, Griffiths AD, Johnson KS, Chiswell DJ, Hudson P, Winter G: Multi-subunit proteins on the surface of filamentous phage: methodologies for displaying antibody (Fab) heavy and light chains. Nucleic Acids Res 1991, 19:4133-4137.

21. Meyers G, Thiel HJ: Molecular characterization of pestiviruses. Adv Virus Res 1996, 47:53-118.

22. Kohl W, Grone A, Moennig V, Herrler G: Expression of the surface glycoprotein E2 of Bovine viral diarrhea virus by recombinant vesicular stomatitis virus. J Gen Virol 2007, 88:157-165.

23. Kuger $P$, Gödecke A, Breunig KD: A mutation in the $Z n$-finger of the GAL4 homolog LAC9 results in glucose repression of its target genes. Nucleic Acids Res 1990, 18:745-751.

24. Mäkelä O, Kaartinen M, Pelkonen JL, Karjalainen K: Inheritance of antibody specificity V. Anti-2-phenyloxazolone in the mouse. J Exp Med 1978, 148:1644-1660.

25. Fiedler U, Phillips J, Artsaenko O, Conrad U: Optimization of scFv antibody production in transgenic plants. Immunotechnology 1997, 3:205-216.

26. Chomczynski P, Sacchi N: Single-step method of RNA isolation by acid guanidinium thiocyanate-phenol-chloroform extraction. Anal Biochem 1987, 162:156-159.

27. Zenke F, Zachariae W, Lunkes A, Breunig KD: Gal80 proteins of Kluyveromyces lactis and Saccharomyces cervisiae are highly conserved but contribute differently to glucose repression of the galactose regulon. Mol Cell Biol 1993, 13:7566-7576.

28. Gurkan C, Symeonides SN, Ellar DJ: High-level production in Pichia pastoris of an anti-p185HER-2 single-chain antibody fragment using an alternative secretion expression vector. Biotechnol Appl Biochem 2004, 39:115-122.

29. Cunha AE, Clemente JJ, Gomes R, Pinto F, Thomaz M, Miranda S, Pinto R, Moosmayer D, Donner P, Carrondo MJ: Methanol induction optimization for scFv antibody fragment production in Pichia pastoris. Biotechnol Bioeng 2004, 86:458-467.

30. Emberson LM, Trivett AJ, Blower PJ, Nicholls PJ: Expression of an anti-CD33 single-chain antibody by Pichia pastoris. I Immunol Methods 2005, 305:135-151.

31. Hu S, Li L, Qiao J, Guo Y, Cheng L, Liu J: Codon optimization, expression, and characterization of an internalizing anti-ErbB2 single-chain antibody in Pichia pastoris. Protein Expr Purif 2006, 47:249-257.

32. Breunig KD, Kuger P: Functional homology between the yeast regulatory proteins GAL4 and LAC9: LAC9-mediated transcriptional activation in Kluyveromyces lactis involves protein binding to a regulatory sequence homologous to the GAL4 protein-binding site. Mol Cell Biol 1987, 7:4400-4406.

33. Rumenapf T, Unger G, Strauss JH, Thiel HJ: Processing of the envelope glycoproteins of pestiviruses. J Virol 1993, 67:3288-3294.

34. Zachariae W, Breunig KD: Expression of the transcriptional activator LAC9 (KIGAL4) in Kluyveromyces lactis is controlled by autoregulation. Mol Cell Biol 1993, 13:3058-3066.
35. Zachariae W: Regulation des Hefetranskriptionsaktivators Lac9. University of Duesseldorf: PhD thesis; 1994.

36. Breunig KD: Multicopy Plasmids Containing the Gene for the Transcriptional Activator LAC9 Are not Tolerated by K. lactis Cells. Curr Genet 1989, 15:143-148.

doi:10.1186/1475-2859-11-112

Cite this article as: Krijger et al: A novel, lactase-based selection and strain improvement strategy for recombinant protein expression in Kluyveromyces lactis. Microbial Cell Factories 2012 11:112.

\section{Submit your next manuscript to BioMed Central and take full advantage of:}

- Convenient online submission

- Thorough peer review

- No space constraints or color figure charges

- Immediate publication on acceptance

- Inclusion in PubMed, CAS, Scopus and Google Scholar

- Research which is freely available for redistribution 